\title{
Fractal analysis of biological wastewater treatment efficiency
}

\author{
Nazira Dzhumagulova ${ }^{1,2}$, Ilya Svetkov $^{2}$, Vladimir Smetanin ${ }^{2}$, and Nguyen Dinh Dap ${ }^{1, *}$ \\ ${ }^{1}$ Moscow State University of Civil Engineering, Yaroslavskoe shosse, 26, Moscow, 129337, Russia \\ ${ }^{2}$ Russian State Agrarian University - Moscow Timiryazev Agricultural Academy, 127550, \\ Timiryazevskaya st. 49, Moscow, Russia
}

\begin{abstract}
The purpose of the present research was to enhance the efficiency of biological wastewater treatment through the direct impact on the metabolism of activated sludge. In the course of research, species and quantitative composition of biological community of activated sludge in aeration tanks during wastewater purification process was studied. Comparative analysis was carried out for linen production wastewater and household sewage. Possible application of biological treatment in linen production was evaluated. Proposals were developed on creation of controllable biological treatment facility. In this paper, biological methods are shown to be efficient for household sewage treatment. Comparative analysis was carried out for linen production wastewater and household sewage. Possible application of biological treatment in linen production was evaluated. Proposals were developed on creation of controllable biological treatment facility.
\end{abstract}

\section{Problem statement}

Until the present, there has been no a quite reliable bioindication system of for the process of biological wastewater treatment. During the process of water purification, the species composition of activated sludge goes through a sequence of changes due to the removal of the corresponding substrates from the sludge liquor. Transition of the biological community from one stage to another is associated with the change in the quantity of nutrients contained in the treated medium, i.e. in case of activated sludge, with BOD values on ashfree basis (per $1 \mathrm{~g}$ ). This process leads to higher species being involved into the community. Depending on the wastewater composition, biological community of activated sludge may consist of a large diversity of unicellular organisms (up to 45 species) with various quantitative distribution of certain species.

As a specific stage, linen production process includes preliminary soaking and scutching of the raw material. Process wastewater from linen soaking contains a wide range of contaminants, most of which are nitrogen compounds. Generally, the wastewater does not contain heavy metals. Wastewater characterized by these types of contaminants can be efficiently treated using biological methods.

\footnotetext{
* Corresponding: nguyendinhdap@gmail.com
} 
In practice, chemical agents (carbonate, bicarbonates, alkalies, etc.) added in order to bind organic acids produced when linen is soaked and treated with sulfuric acid, which is used to accelerate the process and eliminate an unpleasant odor, were proved to be unsuitable [1]. Linen fabric bleaching and dying stages also contribute to contamination of the wastewater. In this case, much more types of contaminants can get into the wastewater. They include chlorine and nitrogen compounds, phosphates, sulphides and synthetic surfactants. For today, one of the most efficient and inexpensive techniques of wastewater purification is biological treatment [2].

In biological treatment, oxidation, cleavage and subsequent removal of organic and inorganic contaminants from the wastewater occurs as a result of vital activity of the biological community. After deactivation, the depleted sludge from this method of wastewater treatment can be used as a filling material in landscape development and land reclamation [3].

The main disadvantage of the biological treatment consists in that it is difficult to implement dynamic process control and adjust the community composition. In order to evaluate the efficiency of biological treatment, multifractal analysis techniques can be used, which allow to conclude on the nature of the processes under study, as well as whether optimum parameters are reached and how stable they are, while providing a high level of sensitivity [4].

\section{Materials and methods research}

Fractal analysis is one of the new techniques for studying complex dynamic systems. It also includes a multifractal dynamics method. Many dynamic processes are readily modeled by fractal curves. Application of fractal dynamic characteristics, primarily fractal dimension, can yield good results in describing the dynamics of such processes [5]. Fractal dimension is essentially a fractional value and it describes the degree of complexity and self-similarity of a curve or a surface. Apart from geometric objects, fractal analysis techniques can also be used for modeling time curves of various parameters of dynamic processes [5].

In literature, the grounds are given for using of threshold values of fractal characteristics to describe processes in their dynamics [5]. Three threshold parameters were revealed, upon reaching of which the process behavior changed. Their values can slightly differ for dynamic processes of various nature, but in most cases they are quite similar. Low threshold value $D_{d}=1,2$ indicates sluggish or no interaction regarding the component under study. Medium responds poorly to the presence of the component which parameters are analyzed in their dynamics. When fractal dimension is about $1,4\left(D_{o}\right)$, the system is in equilibrium state, the interaction is intensive and stable. When fractal dimension $D$ approaches a critical value of 1,7 $\left(D_{k}\right)$, situation for the component becomes unstable, equilibrium is lost, and the system can undergo catastrophic changes, i.e. the value may change by several times over a short period of time (comparing to observation time). This is the basic principle for using fractal dimension fluctuations as a "flag" of catastrophe. In order to find limiting conditions and the condition which is the most favorable for vital activities of biological systems, we can bind these conditions to the fractal parameters above.

As more convenient form of fractal dimension, normalized fractal dimension (fractal temperature) can be used [5]. Similar to physical temperature, which characterizes the degree to which the object is heated, fractal temperature characterizes the dynamics of the process.

Fractal temperature can be derived from fractal dimension using the following expression [5]: 


$$
T_{c}=\left(\frac{1}{2-D}-1\right)
$$

Fractal temperature is based on fractal dimension of a time-dependent process. Fractal dimension describes the nature of the processes occurring in the system and the processes of interaction between the system and the environment [4]. Fractal temperature acts as a parameter that characterizes intensity of exchange interaction with the environment and development of the structure (outflows) of mass and energy redistribution pattern within the system.

\section{Results and discussion}

Time-series data were analyzed in a special software, which determined their fractal dimensions. For clarity, the obtained results can be shown in radar chart (Fig. 1). In this chart, the data on the dynamics of dissimilar processes can be represented within a single metric space - fractal parameter space. By connecting the edges in this chart, we create a geometric shape which allows us to visually estimate the dynamics of a group of processes over time. We also obtain a phase-plane plot of the entire system in the fractal phase space. Circles in the chart correspond to threshold values of fractal temperature $T_{d}, T_{o}, T_{k}$, approaching which indicates a qualitative change of the process under study. Going beyond the critical values of fractal parameters for several components indicates an overall critical condition of the system, and vice versa, contraction of the multifractal phase-plane pattern means an overall decrease of processes intensity in the system and worse stability of the system as a whole.

Methods of fractal analysis allow to evaluate the degree of suppression or development of particular species in the community of microorganisms used for biological wastewater treatment. Based on the results obtained, phase-plane plots were drawn up and analyzed.

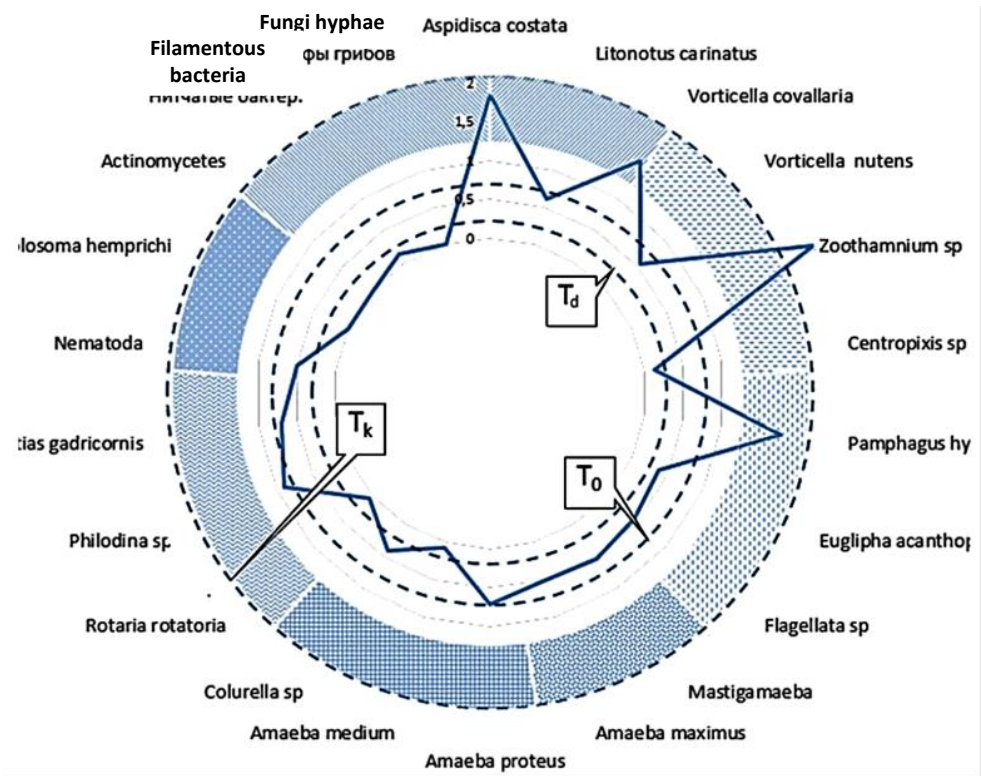

Fig. 1. Multifractal phase-plane plot of dynamics of microorganism species composition in biological treatment community. Microorganism species are shown with hatching 
Fig. 1 shows a multifractal phase-plane plot describing dynamic behavior of species microorganisms composition in the biological community used for biological wastewater treatment. Fractal dimensions of biochemical parameters are increased for infusoria, especially for Zoothamniumsp., which indicates high biological activity of this type of bacteria, temperature conditions favorable to them and possible rapid increase of their population subject to sufficient nutrition. Ameba species demonstrate a moderate activity, while staying in equilibrium state under the current conditions. Some species of bacteria and unicellular organisms, such as thread bacteria, stay inactive, obviously being suppressed.

In the same manner, we can analyze dynamics of physicochemical parameters of water delivered to treatment. The results of this analysis are shown in Fig. 2. The phase-plane plot has quite irregular elongated shape. Especially strained situation is observed for such parameters as Oil Products, total nitrogen and impurities.

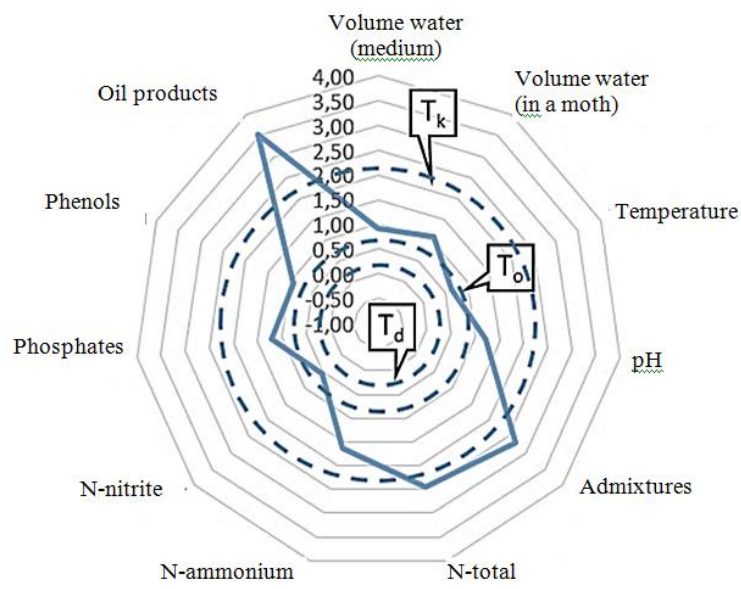

Fig. 2. Multifractal phase-plane plot of dynamics of physicochemical parameters of wastewater delivered to biological treatment

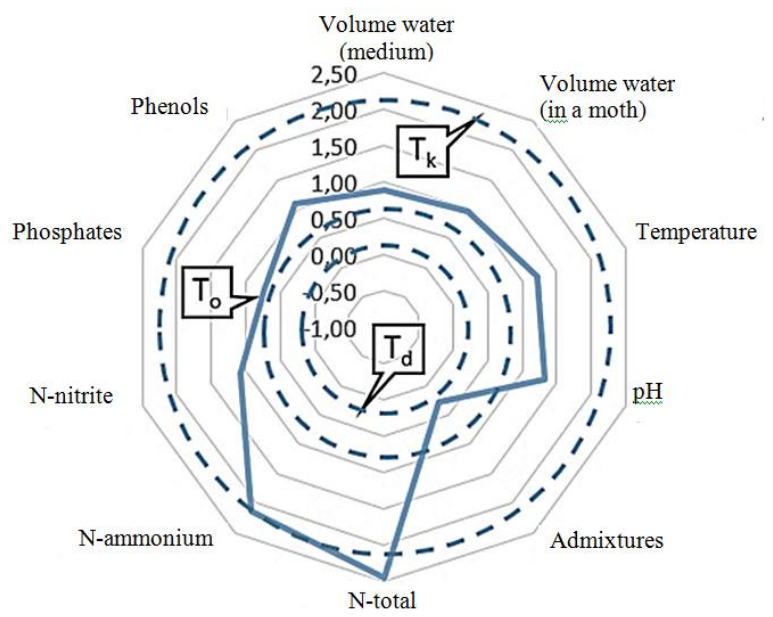

Fig. 3. Multifractal phase-plane plot of dynamics of physicochemical parameters of wastewater after clarification 
Having analyzed a set of hydrochemical parameters of wastewater after clarification (Fig. 3), we can conclude that during clarification solid particles are removed with high efficiency, while almost no effect is observed for dissolved impurities. Dynamic behavior of impurities remained almost the same as for wastewater before treatment.

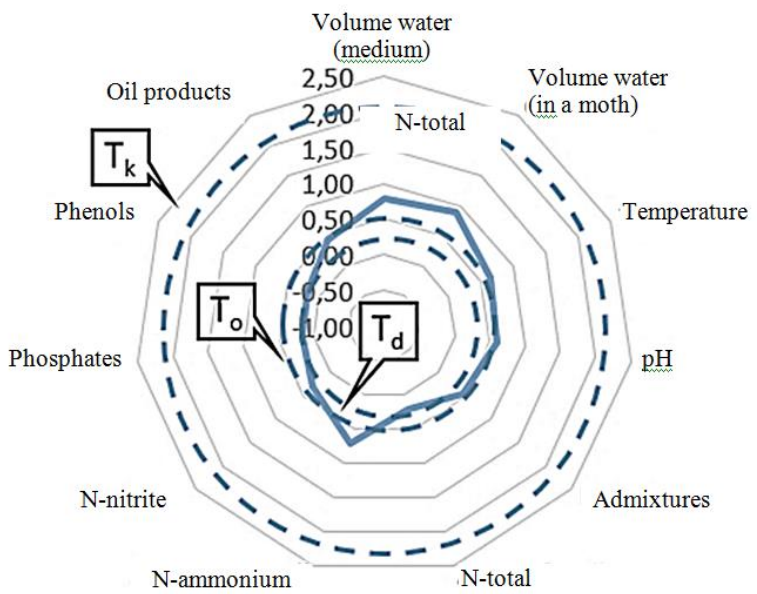

Fig. 4. Multifractal phase-plane plot of dynamics of physicochemical parameters of wastewater after biological treatment

Chemical composition of treated water, as compared to water after clarification, is characterized by lower fractal dimensions of all parameter dynamics and more symmetrical phase-plane patterns with slightly increased chaotic character for nitrogen in the form of ammonium salts. All other parameters remain either in the regions of stability or selfregulation. Thus, a conclusion can be made on the fact that the biological wastewater treatment process is efficient and stable.

One of the main production stages of linen processing is preparation of retted straw from linen raw material. There are several well-known techniques of retted straw preparation - biological, physicochemical and chemical. Biological methods primarily include spreading of linen straw on retteries and warm-water retting. These methods are based on the activity of microorganisms which produce ferments to decompose pectic substances and separate the fibers from adjacent tissues. In chemical method, the fibers are separated due to chemical treatment of linen straw. Regardless of any particular method, the major drawback of the primary stage of linen processing is wastewater equaling $65-75 \%$ wt. of raw material weight. High linen production volumes will result in significantly increased amounts of wastewater. Therefore, to choose the most economically feasible and environmental friendly method of wastewater treatment is a matter of high importance.

The major restriction for application of biological wastewater treatment in textile industry consists in high concentrations of surfactants in the treated water. When concentration of these substances exceeds $20 \mathrm{mg} / \mathrm{l}$, foam generation in aeration tanks is observed along with lower efficiency of treatment. With further growing of surfactants concentration some microorganism species within the community die [6]. Characteristics and concentrations of contaminants in household sewage and textile production wastewater are shown in table 1 [7]. 
Table 1. Characteristics and concentrations of contaminants in household sewage and textile production wastewater

\begin{tabular}{|c|c|c|c|}
\hline Indicators & $\begin{array}{l}\text { Wastewater of } \\
\text { cotton fabric } \\
\text { production }\end{array}$ & $\begin{array}{l}\text { Wastewater of linen } \\
\text { production }\end{array}$ & $\begin{array}{c}\text { Domestic } \\
\text { wastewater }\end{array}$ \\
\hline Water clarity, $\mathrm{cm}$ & $0,1-1,0$ & $1,5-3,5$ & $1-3$ \\
\hline $\mathrm{pH}$ & $9-10$ & $7-8$ & $6,5-8,5$ \\
\hline Suspended substances, $\mathrm{mg} / \mathrm{l}$ & $200-260$ & $200-450$ & $700-1230$ \\
\hline Solid residue, $\mathrm{mg} / \mathrm{l}$ & $600 \ldots 2400$ & $400 \ldots 600$ & $10-800$ \\
\hline Oxidizability, mg/l & 180 & 170 & $200-700$ \\
\hline $\mathrm{BOD}_{20}, \mathrm{mg} \mathrm{O}_{2} / 1$ & $300-400$ & $200-260$ & $10-300$ \\
\hline $\begin{array}{l}\text { Surface active substances, } \\
\mathrm{mg} / \mathrm{l}\end{array}$ & $60 \ldots 80$ & $20 \ldots .25$ & $20 \ldots 30$ \\
\hline Ammonia nitrogen, $\mathrm{mg} / \mathrm{l}$ & $10 \ldots 12$ & $8 \ldots 12$ & $5 \ldots .50$ \\
\hline Chlorides, $\mathrm{mg} / \mathrm{l}$ & $90 \ldots 120$ & $65 \ldots 85$ & $70 \ldots 200$ \\
\hline Sulfates, $\mathrm{mg} / \mathrm{l}$ & $500 \ldots 600$ & $60 \ldots 90$ & $18 \ldots 100$ \\
\hline
\end{tabular}

When comparing the data shown in Table 1, we can conclude that over all categories of parameters and contaminants wastewater from linen production and household sewage are quite similar. Most critical contaminating component for biological treatment is concentration of surfactants [7]. For process operations in cotton production surfactants concentration has increased value, while for linen production wastewater the concentration range does not exceed that for household sewage. This fact determines the applicability of biological treatment methods.

Separate consideration should be given to the issue of treatment of wastewater from dying shops, where certain types of dyes are used and special processing techniques are applied, which result in specific types of contamination.

Thus, one of the major problems of establishing biological wastewater treatment is that the process is difficult to control. Development of biological community varies for media with different compositions. It is difficult to determine the optimum quantity of either biological community loaded into aeration tanks or activated sludge removed from them to subsequent processing and disposal. These tasks can be addressed through application of automatic control system (ACS) which include detectors and control mechanisms [8]. ACS decision-making mechanism can be based on analysis of fractal parameters of main biochemical characteristics being obtained from sensors in real time [9].

\section{Conclusion}

Specific aspects of biological wastewater treatment were considered. Efficiency of biological treatment was determined using fractal analysis. Fractal parameters were used as an indicator of efficiency and stability of the process. Wastewater composition was compared for linen production, cotton production and household sewage. Grounds for application of biological treatment to linen production wastewater were given. Concept of automatic process control system for linen production wastewater treatment was proposed aimed to enhance stability and efficiency of wastewater treatment process.

\section{References}

1. T. Egorova, S. Klenova, E. Sivukhina, Fundamentals of biotechnology (EDP Academy, 385, 2005) 
2. S. Yakovlev, Y. Karelin, Yu. Tender, V. Calico, Sewage and wastewater treatment (EDP Stroyizdat, 510, 2006)

3. O. Kharkina, Effective operation and calculation of structures of biological wastewater treatment (EDP Volggograd-Panorama, 433, 2015)

4. D. Kozlov, A. Nasonov, I. Zhogin, I. Tsvetkov, Water Resources, 2, 259-266 (2017)

5. V. Tsvetkov, I. Tsvetkov, Russian Journal of Earth Sciences, 1, 1-5 (2015)

6. A. Melko, Scientific Journal Advances in current natural sciences, 1, 95-101 (2008)

7. E. Alekseev, Physico-chemical wastewater treament (EDP ASV, 256, 2006)

8. V. Fedorenko, M. Erokhin, V. Balabanov, D. Buligin, I. Golubev, S. Ishchenko, Nanotechnology and nanomaterials in the agricultural sestor (Moscow, Scientific ed., 310, 2011)

9. V. Balabanov, D. Dimitrov, I. Sabirov, Innovations in agriculture, 1, 17-22 (2017) 